ARTICLE

\title{
Attosecond spectroscopy reveals alignment dependent core-hole dynamics in the $\mathrm{ICl}$ molecule
}

Hugo J. B. Marroux (10) 1,2,6凶 , Ashley P. Fidler ${ }^{1,2}$, Aryya Ghosh ${ }^{3}$, Yuki Kobayashi (i) ${ }^{1}$, Kirill Gokhberg ${ }^{3}$,

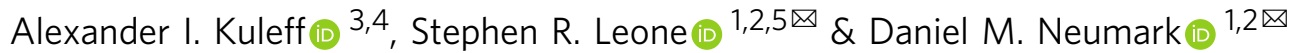

The removal of electrons located in the core shells of molecules creates transient states that live between a few femtoseconds to attoseconds. Owing to these short lifetimes, timeresolved studies of these states are challenging and complex molecular dynamics driven solely by electronic correlation are difficult to observe. Here, we obtain few-femtosecond core-excited state lifetimes of iodine monochloride by using attosecond transient absorption on iodine $4 d^{-1} 6 p$ transitions around $55 \mathrm{eV}$. Core-level ligand field splitting allows direct access of excited states aligned along and perpendicular to the $\mathrm{ICl}$ molecular axis. Lifetimes of $3.5 \pm 0.4 \mathrm{fs}$ and $4.3 \pm 0.4 \mathrm{fs}$ are obtained for core-hole states parallel to the bond and $6.5 \pm$ $0.6 \mathrm{fs}$ and $6.9 \pm 0.6 \mathrm{fs}$ for perpendicular states, while nuclear motion is essentially frozen on this timescale. Theory shows that the dramatic decrease of lifetime for core-vacancies parallel to the covalent bond is a manifestation of non-local interactions with the neighboring $\mathrm{Cl}$ atom of $\mathrm{ICl}$.

\footnotetext{
${ }^{1}$ Department of Chemistry, University of California, Berkeley, CA 94720, USA. ${ }^{2}$ Chemical Sciences Division, Lawrence Berkeley National Laboratory, Berkeley, CA 94720, USA. ${ }^{3}$ Theoretische Chemie, PCI, Universität Heidelberg, Im Neuenheimer Feld 229, 69120 Heidelberg, Germany. ${ }^{4}$ ELI-ALPS, W. Sandner utca 3, Szeged 6728, Hungary. ${ }^{5}$ Department of Physics, University of California, Berkeley, CA 94720, USA. ${ }^{6}$ Present address: Laboratoire de Spectroscopie Ultrarapide (LSU) and Lausanne Centre for Ultrafast Science (LACUS), Ecole Polytechnique Fédérale de Lausanne, ISIC, FSB, Station 6, CH-

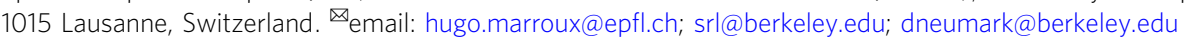


igh-energy photons in the soft $\mathrm{x}$-ray regime access electrons located in core-shells of atoms and molecules. In atoms, excited states resulting from the excitation of a core-shell electron to an outer atomic orbital (core-excited state) decay in tens of femtoseconds or less ${ }^{1,2}$. The decay mechanism, known as Auger decay, is a pure electronic process driven by electronic correlation and consists of the filling of the corevacancy by an outer electron while a secondary electron is emitted in order to conserve energy. The physics of Auger decay in most atomic systems is well established but in molecular systems, complicated symmetry and nuclear effects are anticipated that have so far eluded detailed experimental observation ${ }^{3}$.

Measurement of these excited state lifetimes in molecules through linewidth studies is challenging as lineshape analysis requires consideration of unresolved vibrational structure in addition to lifetime broadening ${ }^{4}$. Attosecond spectroscopy offers the possibility to measure pure electronic dynamics in atoms ${ }^{5}$ and molecules ${ }^{6}$ with unprecedented time resolution. With this time resolution, one can directly measure core-excited-state lifetimes in the time domain ${ }^{7}$ but thus far only isolated atoms $s^{8,9}$ or strong field-related effects in core-excited molecular systems have been investigated ${ }^{10}$. Here, we apply attosecond transient absorption spectroscopy (ATAS) to investigate the decay of core-excited states in the ICl molecule. We find that electronic decay occurs before significant nuclear motion, and that the alignment of the core-level orbital with respect to the internuclear axis has a large effect on lifetimes. These results, observable only by time domain techniques, demonstrate that the decay of molecular core-excited states can be purely electronic processes with timescales strongly affected by molecular structure.

In heteronuclear molecular systems, extreme ultraviolet (XUV) or soft $\mathrm{X}$-ray absorption at an element edge creates a vacancy in a specific atom. The decay mechanisms of the localized core-hole can be divided into local and non-local channels ${ }^{11}$. In the local channels, all the electrons involved originate from the atom bearing the vacancy and these channels are analogous to Auger decay in isolated atoms. In non-local channels, the secondary electron emitted is either localized on another atom or delocalized over multiple atoms. Non-local channels occur via processes that are analogous to interatomic Coulombic decay ${ }^{12}$ or electrontransfer-mediated decay ${ }^{13}$ mechanisms in weakly bound systems, and they can represent a significative portion of the decay process as they often lead to lower energy cations.

The few time-resolved experiments on non-local decay channels obtain lifetimes $>100 \mathrm{fs}^{14-16}$, but those were focused on the study of inner-shell transitions of rare-gas dimers. In covalently bound molecules, the small distances between the atom in which the initial excitation is created and the neighboring atoms can bring the lifetime of the core-hole down to the few or subfemtosecond regime ${ }^{4}$. The resulting large lifetime broadening of the absorption lineshape combined with the complex lineshape of electronic transition in molecules (vibronic progressions, symmetry, etc.) prevent the direct use of frequency-domain techniques to measure molecular core-excited stated lifetimes ${ }^{4}$.

Here, we employ a time-domain approach using ATAS to investigate iodine $6 p \leftarrow 4 d$ core-to-Rydberg transitions around 55 $\mathrm{eV}$ in ICl. Energetically distinct states with iodine core-hole orbitals aligned parallel or perpendicular to the molecular axis are measured $^{17,18}$. Core-hole orbitals aligned parallel to the internuclear axis exhibit significantly shorter lifetimes $(3.5 \pm 0.4$ and $4.3 \pm 0.4 \mathrm{fs})$ compared to core-hole orbitals perpendicular to the molecular axis $(6.5 \pm 0.6$ and $6.9 \pm 0.6 \mathrm{fs})$. During the timescale of these decays, the bond length changes by at most $3.5 \%(0.075 \AA)$, so nuclear motion is minimal. The lifetime dependence on corehole orbital alignment is reproduced in part by ab initio calculations using the Fano-algebraic diagrammatic construction
(ADC)-Stieltjes method ${ }^{19,20}$. Results from the calculation attribute the differences in decay rates to a greater participation of delocalized molecular orbitals (MO) (non-local effect) in the decay of core-excited states aligned along the molecular axis. The observation of excited state decays that are faster than nuclear motion and the dependence of decay rates on orbital alignment opens an uncharted field of investigation exploring electronic molecular decay dynamics using attosecond spectroscopy.

\section{Results}

Static measurement. The absorption spectrum of ICl corresponding to $4 d^{-1} 6 p$ Rydberg excitation on the I atom is collected by spectrally analyzing an isolated attosecond pulse (IAP) transmitted through the sample as shown in Fig. 1a and is reported in Fig. 1b. This spectrum is similar to published spectra of halogen-containing diatomic molecules ${ }^{17,21}$. As shown in Fig. $1 \mathrm{c}$ and deduced from published photoelectron spectrum ${ }^{22}$, the iodine $4 d$ core-levels are split by $1.7 \mathrm{eV}$ due to spin-orbit coupling, and each spin-orbit level has a ligand-field splitting of $0.3 \mathrm{eV}^{22}$. The four peaks in Fig. $1 \mathrm{~b}$ are labeled by $\Omega_{\mathrm{c}}$, the projection of the core hole orbital and spin angular momentum along the internuclear axis ${ }^{23}$. The core-hole orbitals in Fig. 1c are discussed in more detail below. The spectral resolution is better than $50 \mathrm{meV}$ and does not contribute significantly to the lineshapes.

Time-resolved measurement. In ATAS, an IAP covering the iodine $\mathrm{N}_{4,5}$ edge of $\mathrm{ICl}$ is linearly absorbed by the sample and dispersed on a spectrometer as shown on Fig. 1a. Identical to the optical domain, this absorption step creates a macroscopic polarization in the sample that decays with a dephasing time that, in the gas phase, is directly linked to the lifetime of the coreexcited state ${ }^{24}$. The decay of the polarization, termed free induction decay (FID), results in an absorption feature in the IAP. In the time domain, the FID is longer lived than the IAP and can be perturbed by a delayed NIR pulse with sub- 4 fs duration. The perturbed FID induces changes in the absorption lineshapes 25 that are isolated using a shutter to record the IAP spectra with and without the NIR pulse. The transient absorption is then computed using $\Delta A=-\log _{10}\left(I_{\text {on }} / I_{\text {off }}\right)$, reported here in $\Delta \mathrm{mOD}$ (changes in milli-optical density).

By scanning the delay between the IAP and the NIR pulse, core-hole lifetimes can be retrieved from the polarization decay, as has been shown for inner valence excited states of argon and xenon $^{26,27}$ as well as core states of krypton ${ }^{9}$. ATAS was recently applied to core-excited states of methyl iodide, where transitions to Rydberg excited states dominate the spectrum, but no corehole lifetimes were reported ${ }^{10}$.

Figure $1 \mathrm{~d}$ shows the ATAS spectrum of $\mathrm{ICl}$ for various time delays acquired using the experiment described previously ${ }^{28}$ and in the "Methods" section. Here, positive time delays correspond to the XUV pulse arriving before the NIR pulse. The signal is composed of negative transient features at central frequencies of the transitions observed in the static spectrum (Fig. 1b), and positive features on either side of these.

Perturbation of the FID can proceed via laser-induced ionization of the decaying dipole ${ }^{29}$, resonant coupling with a dark state $\mathrm{e}^{30}$, or non-resonant AC stark shifting of the excited state $^{25}$. In the work reported here, single photon ionization is inaccessible with the NIR pulse and the peak power was kept low enough $\left(2 \times 10^{13} \mathrm{~W} / \mathrm{cm}^{2}\right)$ to minimize ionization by strong field processes. The $6 s$ state, which is the principal candidate for resonant coupling with the $6 p$ excited states, is too close in energy $(0.8 \mathrm{eV})$ and cannot be populated by the broadband NIR photon energy $^{17}$. In a study of methyl iodide, Drescher et al. ${ }^{10}$ made 
a

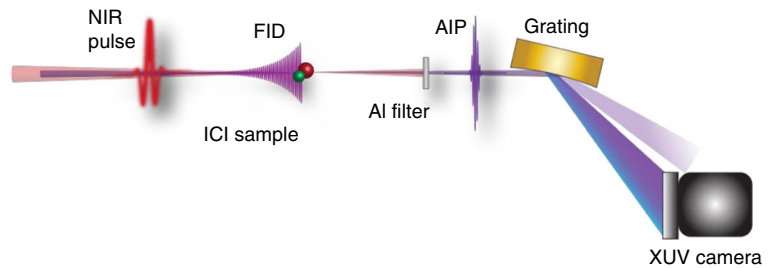

C

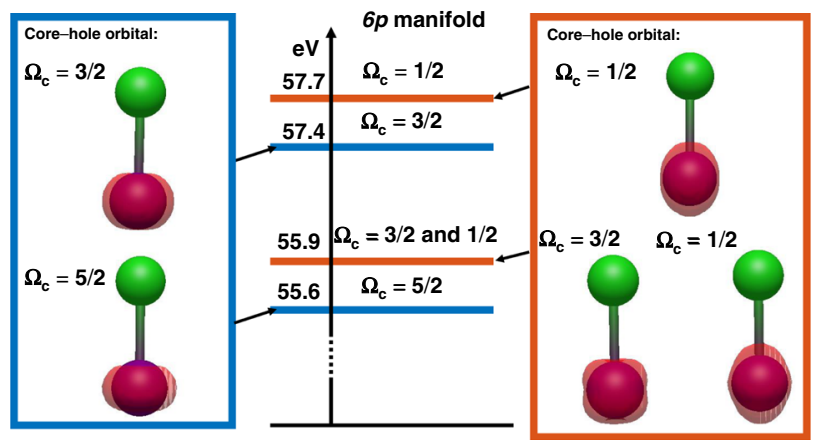

b

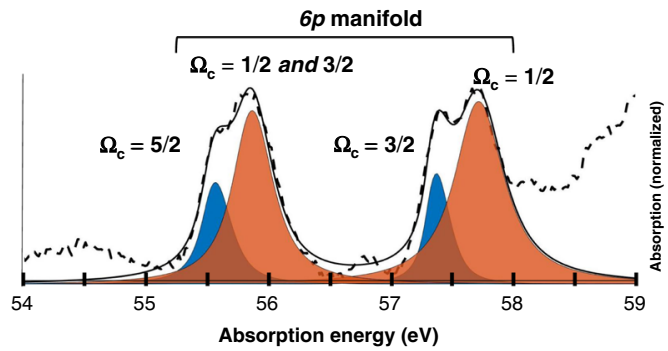

d

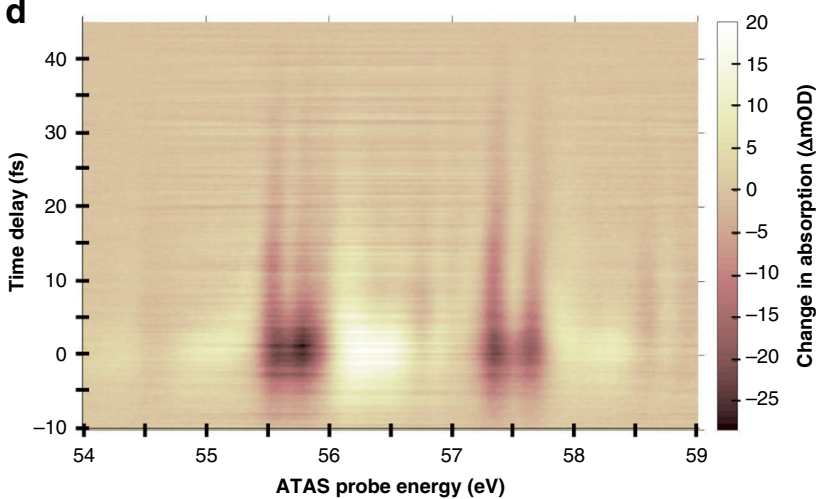

Fig. 1 Attosecond transient absorption of ICI molecules. a Key components of the ATAS experiments. The interaction of the IAP with the sample creates the free induction decay (FID) shown in purple. Delayed NIR pulses perturb the FID, which affects the spectrum of the IAP recorded on the XUV camera and interferes with the incident pulse. The NIR pulse is removed after the sample using an aluminum filter (cf. text for details). Changes in absorption are isolated using an optical shutter to modulate the NIR pulse (not shown). b lodine $\mathrm{N}_{4,5}$ edge absorption of ICl centered around $4 d^{-1} 6 p$ transitions obtained with the described experiment (dashed line). The orange and blue features are the fits to transitions to the indicated $4 d^{-1} 6 p$ core-excited states. The lineshapes include lifetime broadening, finite spectral resolution and vibronic progressions as described in the text. The solid black line is the total fit. c Core-hole orbital electronic density (cf. SM3 for computation details), separated into core-orbitals perpendicular to the molecular (blue box, blue features in b), and orbitals aligned along the molecular axis (orange box, orange features in $\mathbf{b}$ ). The energies are determined based on the fittings to the experimental absorption spectrum. d ATAS spectrum of $\mathrm{ICl}$ at the iodine $\mathrm{N}_{4,5}$ edge in $\triangle \mathrm{mOD}$ (changes in milli-optical density, cf. text for details).

similar observations and concluded that a non-resonant (i.e. Stark shift) interaction is responsible for the transient signal.

In order to confirm that non-resonant coupling of the NIR pulse is the origin of the transient signal, the time-zero transient spectrum has been simulated in Fig. 2a by considering that the NIR pulse induces a shift in the phase $(\Delta \varphi)$ of the FID that is proportional to the ponderomotive energy of the NIR field: $\Delta \varphi(t, \tau)=\int \frac{\left[E_{0}\left(\tau, t^{\prime}\right)\right]^{2}}{4 \omega^{2}} \mathrm{~d} t^{\prime}$, where $E_{0}$ and $\omega$ are the NIR field amplitude and central frequency, respectively $25,30,31$. The timedependent Schrödinger equation is solved considering only the four main resolvable transitions from the core-levels to the $6 p$ Rydberg state. Here the features are uniformly lifetime-broadened to match the experimental spectrum and no vibronic or experimental broadening is taken into account. The main features of the experimental spectrum (in red) are reproduced in the simulation (in gray) confirming that the non-resonant interaction dominates.

The exponential recovery of the negative depletion features depends on the dephasing time of the FID of the considered transition $^{27}$. In the gas phase, dephasing is limited by the population lifetime $\left(T_{1}\right)$ of the excited states so that the FID mentioned earlier is $p(t) \propto \exp \left(-t / 2 T_{1}\right)^{27}$. Hence, the FID decay occurs at half the rate of the population decay. For simplicity, the factor of two between population and dephasing is included in all the time constants reported here and only population lifetimes are discussed (dephasing time constants are reported in Supplementary Table 3 for completeness). Different experimental parameters and checks required to ensure that accurate lifetimes are measured are discussed in the "Methods" section. The kinetic traces yielding lifetimes are obtained by taking lineouts at each of the central frequencies of the absorption features and fitted to an exponential recovery function convoluted with a Gaussian to capture the finite time resolution of the experiment.

The central energies of the four transitions discussed here are determined by fitting the static spectrum with lineshapes obtained by considering expected vibronic progressions, convoluted with the $50 \mathrm{meV}$ spectral resolution and varying the values of the line broadening due to lifetime (cf. SM1 for details). Figure $2 b, c$ then show the kinetic fits at each feature's central frequencies reported at the positions of the arrows in Fig. 2a. In ICl, the decomposition of the static spectrum in Fig. 1b indicates that the features partially overlap, potentially leading to errors in measured lifetimes. This analysis is considered in SM2.

Spectral assignment. The states investigated are located near the iodine $\mathrm{N}_{4,5}$ edge and correspond to $4 d^{-1} 6 p$ core-excited states. The spectral assignment is performed using ab-initio calculations including the spin-orbit coupling induced by the iodine atom. In this context and similarly to other halogen containing diatomic molecules $^{21}, 4 d$ core-levels of iodine are split into five different core-levels shown in Fig. 1c by a combination of ligand field and spin-orbit splitting. The composition of the resulting core holes in terms of the atomic orbitals $\left(d_{x z}, d_{y z}, d_{z^{2}}\right.$, etc.) is reported in SM3 and is similar to results from previous calculations ${ }^{22}$. This decomposition shows that the main components of the $\Omega_{\mathrm{c}}=5 / 2$, $3 / 2$ orbitals in the blue box are the $d_{x^{2}-y^{2}}$ and $d_{x y}$ orbitals, where the $z$-axis is located along the internuclear axis. The high values of the angular momentum projection $(\Lambda=2)$ of these orbitals on the internuclear axis indicate that the electron density of these two orbitals is aligned perpendicular to the molecular axis. On the 

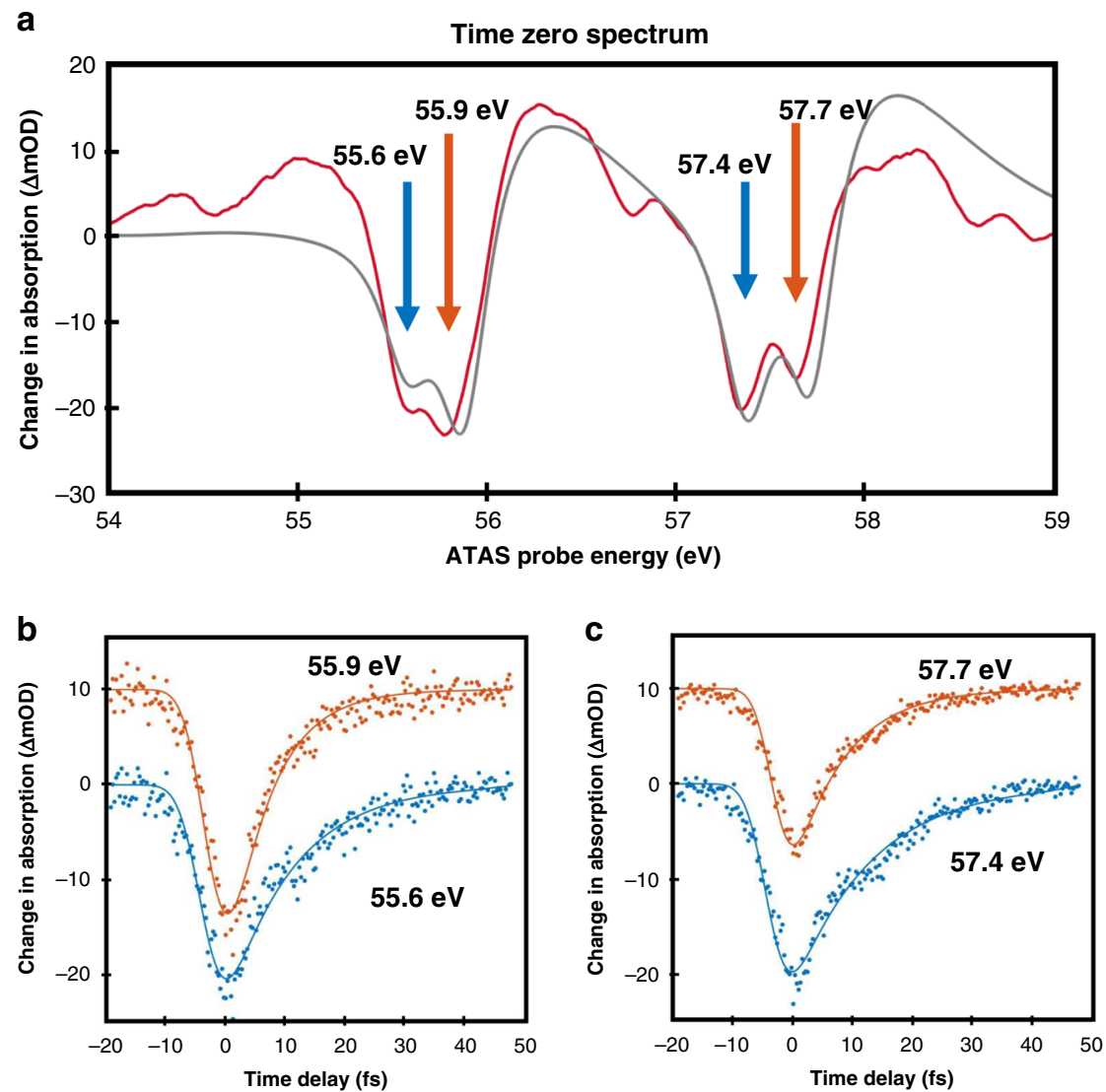

Fig. 2 ATAS spectra simulation and kinetics. a Time zero ATAS experimental (in red) and simulated (in gray) spectra of ICl. The arrows show the positions of the resonances obtained from the fit of the spectrum in Fig. 1b. Kinetic traces of the depletion recovery at the resonance energy are shown as dots in b, c. As described in the text, the kinetic traces at 55.9 and $57.7 \mathrm{eV}$ can directly be linked to the lifetimes of core holes. Traces obtained at 55.6 and $57.4 \mathrm{eV}$ have overlapping contributions as described in the main text and in SM2. The orange traces in $\mathbf{b}, \mathbf{c}$ have been displaced vertically by $10 \Delta \mathrm{mOD}$ for clarity. Solid lines are fits obtained using the convolution of an exponential recovery and gaussian function.

other hand, the three $\Omega_{\mathrm{c}}=3 / 2,1 / 2$ orbitals in the orange box are mainly composed of the $d_{x z}, d_{y z}$ and $d_{z^{2}}$ orbitals, which have angular momentum projection of 1 and 0 indicating an electron density more parallel to the molecular axis.

Experimentally, due to the lineshape broadening of $\sim 130 \mathrm{meV}$ from vibrational effects, and a further $75-180 \mathrm{meV}$ of lifetime broadening (depending on the states), only two types of core-hole alignment are discernible. The two core-orbitals with $\Omega_{\mathrm{c}}=3 / 2,1 /$ 2 in the lower part of the orange box in Fig. $1 \mathrm{c}$ and located at 55.9 $\mathrm{eV}$ are nearly degenerate and are treated together. The three other orbitals can be spectrally isolated and are treated independently.

Nominally, $6 p$ Rydberg orbitals are assigned to $6 p \sigma$ and $6 p \pi$ states. Due to the large radius of Rydberg orbitals, the $6 p \sigma / 6 p \pi$ splitting is too small $(<50 \mathrm{meV})$ compared to the transition linewidths to be resolved. Both orbitals will thus be referred to as $6 p$ without further distinction. Transitions to other Rydberg states, e.g. $6 s$ or $7 p$, are possible, but because of the relatively weak transition dipole moments to these states, transitions to $6 p$ Rydberg states dominate the static absorption spectrum in the spectral region considered ${ }^{17}$.

Molecular dynamics. From the measured lifetimes, the timescales for electronic decay are much shorter than nuclear motion. The core-excited states discussed here are bound and the computed I-Cl stretch frequencies are between 420 and 430 $\mathrm{cm}^{-1}$ depending on the state considered (cf. SM1 for details). Hence, the half-vibrational period is $\sim 40 \mathrm{fs}$ and the nuclear displacement for a half-vibration is $0.34 \AA$, as inferred from the potential energy curves shown in Supplementary Fig. 1a. Depending on the state considered, the timescale for the population lifetime gives a variation on the average internuclear distance $(2.32 \AA)$ of between $1 \%$ and $3.5 \%$ during one time constant of the electronic decay. This leads to the conclusion that the core-hole decays in $\mathrm{ICl}$ are an example of nearly pure electronic molecular dynamics.

Measured lifetimes show substantial dependence on the alignment of the core-hole MO relative to the molecular axis. As shown in Table 1, core-excited states aligned parallel to the covalent bond, i.e. $\Omega_{c}=3 / 2,1 / 2$ in orange in Fig. $1 c$, are 1.6 and 1.9 times shorter-lived than the states aligned perpendicular to the molecular bond $\left(\Omega_{\mathrm{c}}=3 / 2,5 / 2\right.$ in the blue box). A similar effect was computed for the decay of van der Waals dimers $^{32,33}$ and points to a manifestation of a non-local effect on the iodine core-hole states. To confirm the presence of these effects in ICl we consider the decay channels open to the coreexcited states.

The $4 d^{-1} 6 p$ excited state can decay by two types of pathways as represented in Fig. 3: participator channels (channel i in Fig. 3), where the electron in the $6 p$ Rydberg orbital is ionized or moves to a lower-lying orbital, and spectator channels (channels ii and iii in Fig. 3), where only the valence and core-electrons are involved in the decay. Even though participator channels lead to lower energy products, they are known to be minor in atomic iodine ${ }^{34}$ and xenon ${ }^{35}$. Spectator channels are favored in these atoms because of the stronger Coulombic interaction of the coreelectron with valence electrons relative to the electron in the 
Table 1 lodine $4 d^{-1} 6 p$ core-excited staes lifetimes in ICl.

\begin{tabular}{llll} 
Hole orientation & Perpendicular & Parallel & Perpendicular \\
\hline Core-hole character & $\Omega_{c}=5 / 2$ & $\Omega_{\mathrm{c}}=3 / 2,1 / 2$ & $\Omega_{\mathrm{c}}=3 / 2$ \\
Energy (eV) & 55.6 & 55.9 & 57.4 \\
Lifetime (fs) & $6.5 \pm 0.6$ & $3.5 \pm 0.4$ & $6.9 \pm 0.6$ \\
from ATAS measurement & & & $\Omega_{\mathrm{c}}=1 / 2$ \\
\hline
\end{tabular}

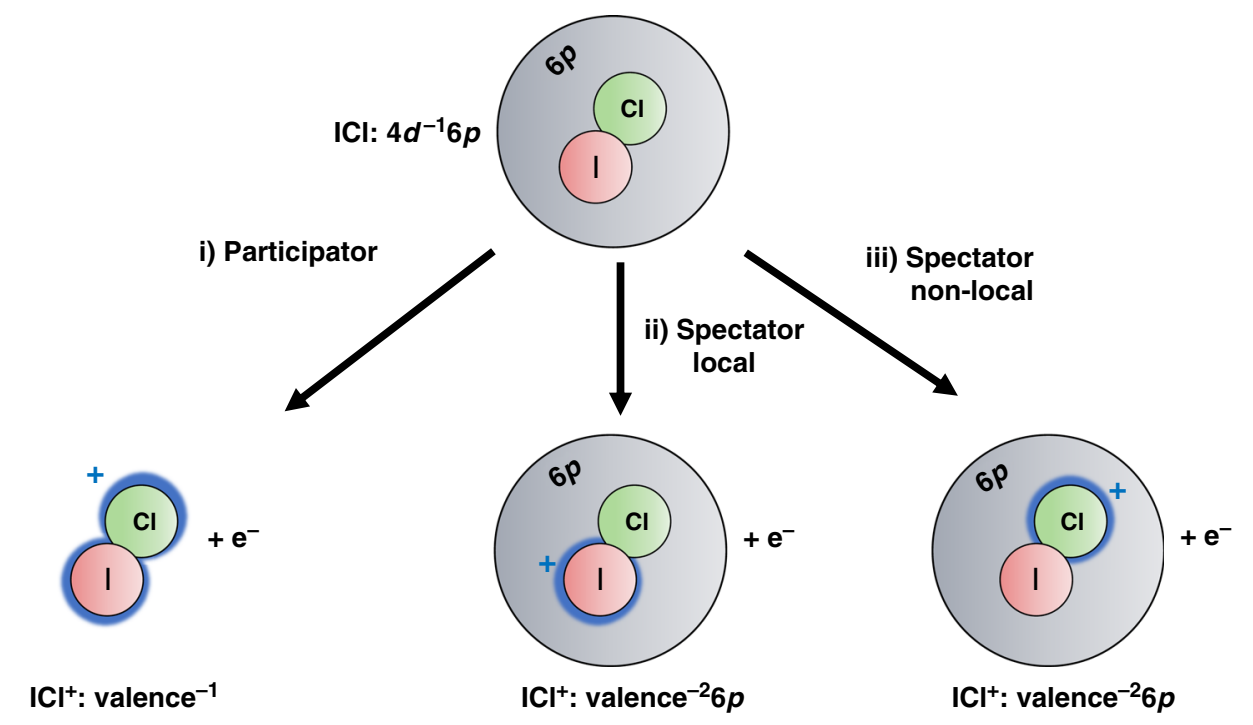

Fig. 3 Open decay channels for $\mathbf{4 d}^{-\mathbf{1}} \mathbf{6 p}$ core-excited state. Channel i represents the participator channels where the electron in the Rydberg $6 p$ orbital (represented in gray) is involved in the electronic decay. The local and non-local channels of the spectator decay, channels ii and iii respectively, lead to a partial charge on the iodine or chlorine atoms, as illustrated by the blue halos.

delocalized $6 p$ Rydberg state. The situation in ICl is similar so the participator channels are not expected to contribute significantly to the core-excited state decay.

To understand the variation of lifetime with core-hole alignment, ab initio calculations of the core-hole partial linewidth were conducted using the Fano-ADC-Stieltjes method as detailed in the "Methods" section 19,20. The calculation yields the partial linewidth associated with each open decay channel, which can be converted to lifetime using $\tau=\hbar / \Gamma$, where $\Gamma$ is the linewidth, and $\tau$ the lifetime. The method relies on a non-relativistic Hamiltonian, so effects such as spin-orbit interaction cannot be reproduced. Decomposition of the orbitals shown in Fig. 1c into $d$-orbitals excluding spin-orbit interactions are shown in SM3.

The calculation of partial linewidths of core-excited states is difficult due to the complexity of the final state manifold. However, as core-hole relaxation is expected to be dominated by spectator decay, it is a reasonable approximation to consider the core ionized molecule rather than the $4 d^{-1} 6 p$ state of the neutral. Therefore, we computed partial linewidths of ICl cations with a hole in the $4 d$ orbital. In these conditions, the three cationic states considered are the ${ }^{2} \Sigma,{ }^{2} \Pi$, and ${ }^{2} \Delta$ states, which correspond to the ionization limits of the $4 d \sigma n p, 4 d \pi n p$, and $4 d \delta n p$ Rydberg series, respectively.

At the energy considered, 48 channels are open (cf. SM4). The decay channel showing the largest variation of partial linewidth with the type of initial core-hole is displayed in Fig. 4a. Given that the calculation considers core-excited cations as initial states, decay products are doubly charged with the two holes located in available valence MOs. For the channel considered in Fig. $4 \mathrm{a}$, the two holes of this decay product are located in the same valence
MO shown in the inset of Fig. 4a. For this final state, partial linewidths for the ${ }^{2} \Sigma,{ }^{2} \Pi$, and ${ }^{2} \Delta$ core-holes are $14.1,5.3$, and $2.3 \mathrm{meV}$, respectively. The evolution of the linewidths with the core-hole types shows the decrease of the contribution of this final state to the core-hole decay. The main atomic orbital contributing to the empty MO shown is the $\mathrm{Cl} 3 p$ ( $80 \%$ of the total orbital) so the contribution of non-local effects on the iodine core-hole decay is major for this channel. This highlights the role of non-local effects and core-orbital alignment on the core-hole decay.

In order to compare experimental and computational results, all the decay channels have been considered and the MO of all the product and partial widths are shown in SM4. The total linewidths of each of the hole types are obtained by summing the partial linewidths of all open decay channels.

The lifetimes computed from the total widths are shown in Fig. $4 \mathrm{~b}$ and are approximately twice as short as the experimental ones. This is due to a known bias in the computational method and consistent with other known $\operatorname{cases}^{36-38}$. Despite the approximations in the calculations (no spin-orbit interaction, considering the molecular cation, etc.), the relative simulated lifetimes capture the dependence on the core-hole alignment at various internuclear distances. At the equilibrium distance, $\left(R_{\mathrm{eq}}=2.32 \AA\right)^{39}$, the computed lifetime of the ${ }^{2} \Sigma$ state is 1.25 times shorter than the lifetimes of ${ }^{2} \Pi$ and ${ }^{2} \Delta$. This can be compared to the ratio of the $\Omega_{c}=3 / 2,1 / 2$ core holes lifetime at $55.9 \mathrm{eV}$ and the $\Omega_{\mathrm{c}}=5 / 2$ core-hole lifetime at $55.6 \mathrm{eV}$ measured by ATAS of 1.9. The lifetimes computed for the cations in the ${ }^{2} \Pi$ and ${ }^{2} \Delta$ states at the equilibrium bond distance are similar despite the differences in the core-hole wavefunction alignments. This may reflect inaccuracies in the lifetime calculation due to the 
a

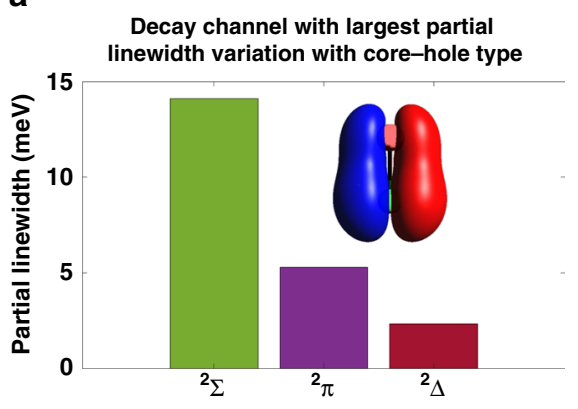

b

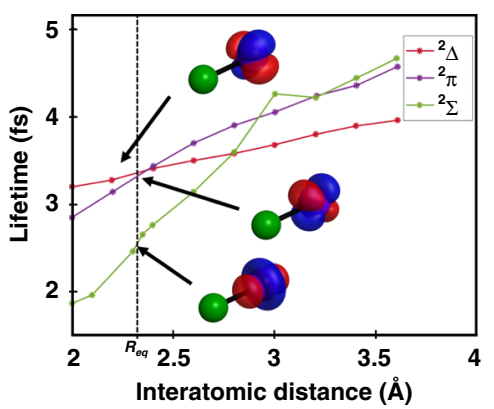

C At equilibrium:

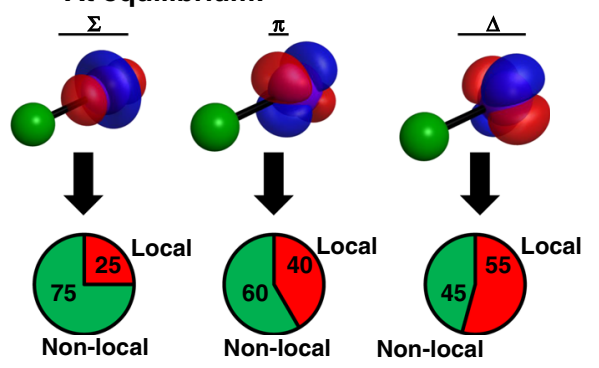

Fig. 4 Origin of the lifetime variation. a Partial linewidths of each of the core-hole types for the decay channel showing the largest partial linewidth variation. For this channel, the two valence holes of the decay product are located in the same MO shown in the inset. $\mathbf{b}$ Calculated core-hole lifetime of the cation for the three types of core-hole orbitals at various internuclear distances. Conversion between linewidth and lifetime is obtain using $\tau=\hbar / \Gamma$, where $\Gamma$ is the linewidth, and $\tau$ the lifetime. The equilibrium distance (2.32 $\AA$ ) is shown by the dotted line. c Contribution of the local and non-local decay chanels at the equilibrium internuclear distance.

neglect of spin-orbit effects or consideration of the core-excited cation rather than the neutral species.

Computations consider the effect of the neighboring chlorine atom on the measured iodine core-hole lifetimes. In order to classify the valence delocalized orbitals into local and non-local channels, the final state empty MOs bearing the charges are projected onto atomic orbitals. Weights of the two types of channels are shown for each core-hole type at the equilibrium distance in Fig. $4 \mathrm{c}$. While the ${ }^{2} \Pi$ and ${ }^{2} \Delta$ coreorbitals show similar contributions of local and non-local channels, the ${ }^{2} \sum$ core-hole shows a major contribution of nonlocal effects.

\section{Discussion}

Molecular core-excited states dynamics of iodine monochloride (ICl) following $6 p \leftarrow 4 d$ core-to-Rydberg excitation are measured using ATAS. We show that this technique gives a direct access to lifetimes of the core-hole and allows one to follow the molecular decay in real time. Four molecular core-excited state lifetimes between 3.5 and $6.9 \mathrm{fs}$ are reported depending on the core-hole character.

During the decay of the core-excited states with $4 d^{-1} 6 p$ character the nuclei move by $<3.5 \%$ of their internuclear distance. Following on the ever-growing interest in charge migration 40,41 and photoionization time delays ${ }^{42}$, this investigation shows not only that the molecular dynamics of core states can be investigated by attosecond spectroscopy, but that their decays can be a nearly pure electronic decay process.

In this regime of molecular dynamics, core-excited state lifetimes depend on the core-hole orbital alignment with respect to the molecular axis. Core-hole orbitals with $\Omega_{\mathrm{c}}=3 / 2$ and $1 / 2$ and parallel to the molecular axis exhibit shorter lifetimes than orbitals with $\Omega_{c}=5 / 2$ and $3 / 2$ and perpendicular to the molecular axis. Calculations confirm this effect and attribute it to the larger contribution of non-local effects on the decay due to the presence of the nearby chlorine atoms for hole orbitals aligned along the molecular axis.

Molecular core-levels are often considered to be little influenced by the valence structure or molecular environments and are often replaced by pseudopotentials in electronic structure calculations in order to simplify relativistic effects ${ }^{43}$. Previous studies showed that core-level spectra can be non-degenerate due to ligand-field splitting ${ }^{22}$. This time-resolved study shows that their lifetimes are also greatly influenced by the molecular structure. This opens questions on the effect of lower molecular symmetry, ligand electronegativity, solvation environment, etc. on core- excited state lifetimes. These topics relate to a wide range of fields such as chemistry and can now be addressed using attosecond spectroscopy.

\section{Methods}

ATAS experiment. The laser setup was reported elsewhere ${ }^{30}$. Briefly, a carrier envelope phase stable Ti:Sapphire oscillator (Femtolaser, Rainbow) and multipass amplifier are used to produce carrier envelope stabilized pulses $(1.8 \mathrm{~mJ}, 1$ $\mathrm{kHz}, 25 \mathrm{fs}$ at $780 \mathrm{~nm}$ ). The laser pulses are spectrally broadened in a stretched hollow-core fiber of $2 \mathrm{~m}$ length and a $500 \mu \mathrm{m}$ inner diameter (few-cycle Inc.) filled with Neon gas. Pulses are compressed using seven pairs of double angled chirped mirrors (Ultrafast Innovations, PC70) and a $3 \mathrm{~mm}$-thick ammonium dihydrogen phosphate crystal to correct for third-order dispersion ${ }^{44}$. The compressed beam is separated by a 70/30 broadband beam splitter (LAYERTEC) and directed toward the high-order harmonic generation (HHG) cell and probe beam, respectively. IAPs are generated by focusing the $3.7 \mathrm{fs}$ long pulse (1.4 optical cycle) into a gas cell with flowing argon using a $f=500 \mathrm{~mm}$ concave mirror ${ }^{44}$. The driving field characterization was done using a commercially available dispersion scan, i.e. d-scan (Sphere Ultrafast Photonics) and the results are shown in SM5. The isolated character of the attosecond pulse was established by confirming that the spectrum was continuous and showed a strong variation with the laser carrier envelope phase of the driving pulse, based on previous streaking measurements ${ }^{44}$.

The driving NIR field for the HHG is separated from the IAPs using a $200 \mathrm{~nm}$ thick aluminum filter supported on a mesh (Lebow). The IAP pulses are refocused toward the sample gas cell using a gold-coated toroidal mirror. The delayed NIR pulse is recombined with the IAP between the toroidal mirror and the target cell using an annular mirror. The delayed NIR pulse is focused using a silver-coated $f=1000 \mathrm{~mm}$ concave mirror. After the target cell, the NIR probe pulse is then removed using an aluminum filter similar to that used to separate the HHG. An aberration-corrected concave grating (Hitachi, part number 001-0640) is used to disperse the light onto a CCD camera (Princeton Instrument, Pixis) ${ }^{45}$. The experiment is conducted using the diffraction grating in second order and the spectral resolution was determined to be $50 \mathrm{meV}$ at $65 \mathrm{eV}$ by fitting of the corelevel transitions in xenon.

The ICl sample was purchased from Sigma Aldrich and used without further purification. Adequate sample density was achieved by heating the sample container and gas lines to $40^{\circ} \mathrm{C}$ using heat tapes. Transient absorption spectra were obtained by collecting the IAP spectrum with $\left(I_{\text {on }}\right)$ and without the delayed NIR beam $\left(I_{\text {off }}\right)$ (modulated using a mechanical shutter) and computed using $\Delta A=-\log \left(\frac{I_{\text {on }}}{I_{\text {off }}}\right)$.

Core-hole lifetime measurement. To reduce systematic errors in lifetime measurements due to low spectral resolution, inducing an early cancellation of the depletion feature by nearby positive features, static and time-resolved data were collected using the second order of the spectrometer diffraction grating reaching a spectral resolution of $50 \mathrm{meV}$. Power dependences and discussion of the spectral resolution are detailed in the SM6 to verify that the measurements accurately report core-hole lifetimes. To further confirm the lifetime measurement, the lifetime of core-excited xenon following excitation of its $6 p<4 d$ transition was measured using ATAS under the same laser power, pressure and spectral resolution conditions as those used in the ICl experiments. A lifetime of $5.9 \pm 0.7 \mathrm{fs}$ is measured in these conditions, in good agreement with a previous estimate from linewidth measurements, which indicated a lifetime of $6.2 \pm 0.2 \mathrm{fs}^{46}$. (cf. SM7 for spectrum and kinetic traces). 
Fano-ADC-Stieltjes method. Ab initio calculations of the core-hole lifetime were conducted using the Fano-ADC-Stieltjes method ${ }^{19,20}$ In the Fano-ADC-Stieltjes method, natural linewidths $(\Gamma)$ are obtained by separately constructing the continuum composed of the decaying state and the leaving electron $\left(\chi_{\beta, \varepsilon}\right)$, the bound initial state $(\Phi)$, and the coupling between the two. The width is given by the golden rule-like expression, where the coupling moments are summed over all open decay channels.

$$
\Gamma=2 \pi \sum_{\beta}\left|\left\langle\Phi|\hat{H}| \chi_{\beta, \varepsilon}\right\rangle\right|^{2}
$$

The width is then converted to lifetime following the uncertainty principle $(\tau=\hbar / \Gamma)$.

As discussed in the main text, the decay of core-excited states considered mainly occurs via spectator mechanisms where the electron in the $6 p$ Rydberg orbital does not participate. To simplify the calculation, core-ionized cations were, therefore, considered instead of core-excited ones. Moreover, the non-relativistic Hamiltonian has been used to construct the initial and final states and, thus, the spin-orbit interactions have been neglected. In these approximations, three core ionized states are considered: the ${ }^{2} \Sigma,{ }^{2} \Pi$, and ${ }^{2} \Delta$, corresponding to the ionization limits of the $4 d \sigma n p$, $4 d \pi n p$, and $4 d \delta n p$ Rydberg series, respectively.

\section{Data availability}

Data are available on request from the authors.

Received: 21 February 2020; Accepted: 13 October 2020;

Published online: 16 November 2020

\section{References}

1. Chattarji, D. The Theory of Auger Transitions (Academic Press Inc, Ed., New York, 1976).

2. Föhlisch, A. et al. Direct observation of electron dynamics in the attosecond domain. Nature 436, 373-376 (2005).

3. Eroms, M., Vendrell, O., Jungen, M., Meyer, H.-D. \& Cederbaum, L. S. Nuclear dynamics during the resonant auger decay of water molecules. J. Chem. Phys. 130, 154307 (2009).

4. Püttner, R. et al. Si 1s -1, 2s -1 and 2p -1 Lifetime broadening of SiX 4 (X=F, $\mathrm{Cl}, \mathrm{Br}, \mathrm{CH} 3)$ molecules: $\mathrm{SiF} 4$ anomalous behaviour reassessed. Phys. Chem. Chem. Phys. 21, 8827-8836 (2019).

5. Gruson, V. et al. Attosecond dynamics through a fano resonance: monitoring the birth of a photoelectron. Science (80-.) 354, 734-738 (2016)

6. Kraus, P. M. et al. Measurement and laser control of attosecond charge migration in ionized iodoacetylene. Science (80-.) 350, 790 LP-790795 (2015).

7. Ramasesha, K., Leone, S. R. \& Neumark, D. M. Real-time probing of electron dynamics using attosecond time-resolved spectroscopy. Annu. Rev. Phys. Chem. 67, 41-63 (2016).

8. Uiberacker, M. et al. Attosecond real-time observation of electron tunnelling in atoms. Nature 446, 627-632 (2007).

9. Hütten, K. et al. Ultrafast quantum control of ionization dynamics in krypton. Nat. Commun. 9, 719 (2018)

10. Drescher, L. et al. State-resolved probing of attosecond timescale molecular dipoles. J. Phys. Chem. Lett. 10, 265-269 (2019).

11. Hergenhahn, U. Interatomic and intermolecular coulombic decay: the early years. J. Electron Spectros. Relat. Phenom. 184, 78-90 (2011).

12. Cederbaum, L. S., Zobeley, J. \& Tarantelli, F. Giant intermolecular decay and fragmentation of clusters. Phys. Rev. Lett. 79, 4778 (1997).

13. Zobeley, J., Santra, R. \& Cederbaum, L. S. Electronic decay in weakly bound heteroclusters: energy transfer versus electron transfer. J. Chem. Phys. 115, 5076-5088 (2001).

14. Schnorr, K. et al. Time-resolved measurement of interatomic coulombic decay in. Phys. Rev. Lett. 111, Ne2 (2013).

15. Takanashi, T. et al. Time-resolved measurement of interatomic coulombic decay induced by two-photon double excitation of Ne2. Phys. Rev. Lett. 118, 033202 (2017).

16. Mizuno, T. et al. Time-resolved observation of interatomic excitation-energy transfer in argon dimers. J. Chem. Phys. 146, 104305 (2017).

17. Brion, C. E., Dyck, M. \& Cooper, G. Absolute photoabsorption cross-sections (oscillator strengths) for valence and inner shell excitations in hydrogen chloride, hydrogen bromide and hydrogen iodide. J. Electron Spectros. Relat. Phenom. 144-147, 127-130 (2005)

18. Olney, T. N., Cooper, G. \& Brion, C. E. Quantitative studies of the photoabsorption (4.5-488 EV) and photoionization (9-59.5 EV) of methyl iodide using dipole electron impact techniques. Chem. Phys. 232, 211-237 (1998).

19. Santra, R. \& Cederbaum, L. S. Non-Hermitian electronic theory and applications to clusters. Phys. Rep. 368, 1-117 (2002).
20. Averbukh, V. \& Cederbaum, L. S. Ab initio calculation of interatomic decay rates by a combination of the Fano Ansatz, Green's-function methods, and the stieltjes imaging technique. J. Chem. Phys. 123, 204107 (2005).

21. Johnson, J., Cutler, J. N., Bancroft, G. M., Hu, Y. F. \& Tan, K. H. Highresolution photoabsorption and photoelectron spectra of bromine-containing molecules at the $\mathrm{Br} 3 d$ edge: the importance of ligand field splitting. J. Phys. B 30, 4899 (1997).

22. Cutler, J. N., Bancroft, G. M. \& Tan, K. H. Ligand-field splittings and corelevel linewidths in I $4 d$ photoelectron spectra of iodine molecules. J. Chem. Phys. 97, 7932 (1992).

23. Lefebvre-Brion, H., Giusti-Suzor, A. \& Rașeev, G. Theoretical Study of the spin-orbit autoionization in molecules application to the HI photoionization spectrum. J. Chem. Phys. 83, 1557-1566 (1985).

24. Beck, A. R. et al. Attosecond transient absorption probing of electronic superpositions of bound states in neon: detection of quantum beats. New J. Phys. 16, 113016 (2014).

25. Wu, M., Chen, S., Camp, S., Schafer, K. J. \& Gaarde, M. B. Theory of strongfield attosecond transient absorption. J. Phys. B 49, 062003 (2016).

26. Wang, H. et al. Attosecond time-resolved autoionization of argon. Phys. Rev Lett. 105, 143002 (2010).

27. Bernhardt, B. et al. High-spectral-resolution attosecond absorption spectroscopy of autoionization in xenon. Phys. Rev. A 89, 023408 (2014).

28. Marroux, H. J. B., Fidler, A. P., Neumark, D. M. \& Leone, S. R. Multidimensional spectroscopy with attosecond extreme ultraviolet and shaped near-infrared pulses. Sci. Adv. 4, eaau3783 (2018).

29. Kaldun, A. et al. Observing the ultrafast buildup of a fano resonance in the time domain. Science (80-.) 354, 738-741 (2016).

30. Cao, W., Warrick, E. R., Neumark, D. M. \& Leone, S. R. Attosecond transient absorption of argon atoms in the vacuum ultraviolet region: line energy shifts versus coherent population transfer. New J. Phys. 18, 013041 (2016).

31. Ott, C. et al. Lorentz meets Fano in spectral line shapes: a universal phase and its laser control. Scence (80-) 340, 716-720 (2013).

32. Kryzhevoi, N. V., Averbukh, V. \& Cederbaum, L. S. High activity of helium droplets following ionization of systems inside those droplets. Phys. Rev. B 76, 94513 (2007)

33. Gokhberg, K., Kopelke, S., Kryzhevoi, N. V., Kolorenč, P. \& Cederbaum, L. S. Dependence of interatomic decay widths on the symmetry of the decaying state analytical expressions and ab initio results. Phys. Rev. A 81, 013417 (2010).

34. Nahon, L. \& Morin, P. Experimental study of Rydberg states excited from the d shell of atomic bromine and iodine. Phys. Rev. A 45, 2887 (1992).

35. Southworth, S. et al. Electron-spectroscopy study of inner-shell photoexcitation and ionization of Xe. Phys. Rev. A 28, 261 (1983).

36. Stumpf, V., Brunken, C. \& Gokhberg, K. Impact of metal ion's charge on the interatomic Coulombic decay widths in microsolvated clusters. J. Chem. Phys. 145, 104306 (2016).

37. Trinter, F. et al. Evolution of interatomic Coulombic decay in the time domain. Phys. Rev. Lett. 111, 093401 (2013).

38. Ouchi, T. et al. Interatomic Coulombic decay following Ne $1 s$ Auger decay in NeAr. Phys. Rev. A 83, 053415 (2011).

39. Herbst, E. \& Steinmetz, W. Dipole moment of ICI. J. Chem. Phys. 56, 5342-5346 (1972).

40. Calegari, F. et al. Ultrafast electron dynamics in phenylalanine initiated by attosecond pulses. Science (80-) 346, 336-339 (2014).

41. Kuleff, A. I. \& Cederbaum, L. S. Ultrafast correlation-driven electron dynamics. J. Phys. B 47, 124002 (2014).

42. Beaulieu, S. et al. Attosecond-resolved photoionization of chiral molecules. Science (80-) 358, 1288-1294 (2017).

43. Dolg, M. Effective core potentials. Mod. Methods Algorithms Quantum Chem. 1, 479-508 (2000)

44. Timmers, H. et al. Generating high-contrast, near single-cycle waveforms with third-order dispersion compensation. Opt. Lett. 42, 811-814 (2017).

45. Wang, X., Chini, M., Cheng, Y., Wu, Y. \& Chang, Z. In situ calibration of an extreme ultraviolet spectrometer for attosecond transient absorption experiments. Appl. Opt. 52, 323-329 (2013).

46. Penent, F. et al. Multielectron spectroscopy: the xenon $4 d$ hole double auger decay. Phys. Rev. Lett. 95, 83002 (2005).

\section{Acknowledgements}

The authors acknowledge Lorenz Cederbaum and Romain Geneaux for valuable discussions. H.J.B.M., A.P.F., D.M.N., and S.R.L. acknowledge the Director, Office of Science, Office of Basic Energy Sciences through the Atomic, Molecular, and Optical Sciences Program of the Division of Chemical Sciences, Geosciences, and Biosciences of he U.S. Department of Energy at Lawrence Berkeley National Laboratory under contract no. DE-AC02-05CH11231. A.P.F. acknowledges funding from the NSF Graduate Research Fellowship Program. Y.K., D.M.N., S.R.L., and A.I.K. acknowledge support 
from the U.S. Army Research Office (ARO) (No. W911NF-14-1-0383). Y.K., and S.R.L. acknowledge support from the National Science Foundation (NSF) (CHE-1660417 and 1951317) for absorption spectra calculations Y.K. also acknowledges financial support from the Funai Overseas Scholarship. A.G., K.G., and A.I.K. acknowledge the support by the European Research Council (ERC) under the Advanced Investigator Grant No. 692657 .

\section{Author contributions}

H.J.B.M., A.P.F., D.M.N., and S.R.L. designed the experiment. H.J.B.M. and A.P.F. performed the data collection. A.G., K.G., and A.I.K. performed the core-hole lifetime calculations and Y.K. performed the absorption spectra calculations. H.J.B.M., D.M.N., and S.R.L. wrote the manuscript.

\section{Competing interests}

The authors declare no competing interests.

\section{Additional information}

Supplementary information is available for this paper at https://doi.org/10.1038/s41467 020-19496-0.

Correspondence and requests for materials should be addressed to H.J.B.M., S.R.L. or D.M.N.
Peer review information Nature Communications thanks Hans Jakob Wörner and the other, anonymous, reviewer(s) for their contribution to the peer review of this work. Peer reviewer reports are available.

Reprints and permission information is available at http://www.nature.com/reprints

Publisher's note Springer Nature remains neutral with regard to jurisdictional claims in published maps and institutional affiliations.

(c) (i) Open Access This article is licensed under a Creative Commons Attribution 4.0 International License, which permits use, sharing, adaptation, distribution and reproduction in any medium or format, as long as you give appropriate credit to the original author(s) and the source, provide a link to the Creative Commons license, and indicate if changes were made. The images or other third party material in this article are included in the article's Creative Commons license, unless indicated otherwise in a credit line to the material. If material is not included in the article's Creative Commons license and your intended use is not permitted by statutory regulation or exceeds the permitted use, you will need to obtain permission directly from the copyright holder. To view a copy of this license, visit http://creativecommons.org/ licenses/by/4.0/.

(C) The Author(s) 2020 